\title{
In Not Only Vertebroplasty but Also Kyphoplasty, the Resolution of Vertebral Deformities Depends on Vertebral Mobility
}

\author{
K. Yokoyama, M. Kawanishi, M. Yamada, H. Tanaka, Y. Ito, M. Hirano, and T. Kuroiwa
}

\begin{abstract}
BACKGROUND AND PURPOSE: It has not been clarified whether the postoperative resolution of vertebral deformities achieved by KP are superior to those achieved by VP. We compared the outcomes of KP and VP, taking into account the presence of vertebral mobility, to determine whether the balloon inflation in KP may contribute to postoperative resolution of the vertebral deformities in patients with VCFs.

MATERIALS AND METHODS: The subjects had 34 vertebral bodies treated by VP, and 43 vertebral bodies treated by KP. In all patients, preoperative dynamic imaging was performed to assess the presence of vertebral mobility. First, the vertebral height restoration and kyphotic change were compared between the VP and KP groups. Then, the patients were further divided into 2 groups on the basis of the presence of vertebral mobility, and they were compared within and between the VP and KP groups in relation to the presence of vertebral mobility.

RESULTS: Overall, no significant differences were observed in either the vertebral height restoration or kyphotic change between the VP and KP groups $(P>$.20). Preoperative dynamic imaging identified 19 vertebral bodies each with vertebral mobility in the VP (56\%) and KP groups $(44 \%)(P=.21)$. Within the VP and KP groups, the vertebral height restoration and kyphotic changes were significantly better in patients with vertebral mobility than in those without $(P<.01)$. There were no significant differences between the 2 treatment groups after adjustment for the presence of vertebral mobility $(P>.30)$.
\end{abstract}

CONCLUSIONS: In both the VP and KP groups, the vertebral height restoration and kyphotic change largely depended on the preoperative vertebral mobility. The use of the balloon itself contributed little to resolution of the vertebral deformities.

ABBREVIATIONS: $\mathrm{KP}=$ kyphoplasty; $\mathrm{PMMA}=$ polymethylmethacrylate; $\mathrm{VAS}=$ Visual Analog Scale; $\mathrm{VCF}=$ vertebral compression fracture; $\mathrm{VP}=$ vertebroplasty

B alloon kyphoplasty is now considered as minimally invasive and effective as conventional percutaneous vertebroplasty for rapid pain relief in patients with painful vertebral compression fractures resistant to conservative treatment. ${ }^{1-9} \mathrm{KP}$ is a treatment procedure that was developed with the expectation that it would provide better resolution of vertebral deformities than VP because of the insertion and inflation of a balloon within the collapsed vertebral body. Although some reports show that the postoperative height restoration and kyphotic changes achieved by KP are significantly superior to those achieved by $\mathrm{VP},{ }^{10-13}$ others report scarce differences in these outcomes between balloon KP and

Received September 10, 2012; accepted after revision October 16.

From the Department of Neurosurgery (K.Y., M.K., M.Y., H.T., Y.I., M.H.), Takeda General Hospital, Fushimi, Kyoto, Japan; and Departments of Neurosurgery and Surgical Pathology (T.K.), Osaka Medical College, Takatsuki, Osaka, Japan.

Please address correspondence to Kunio Yokoyama, MD, Department of Neurosurgery, Takeda General Hospital, 28-1, Ishidamoriminami-machi, Fushimi, Kyoto

City, Kyoto 601-1495, Japan; e-mail: neu100@poh.osaka-med.ac.jp

http://dx.doi.org/10.3174/ajnr.A3424
$\mathrm{VP},{ }^{14-16}$ and no consensus has yet been reached. It has been reported that the presence of vertebral mobility associated with pseudoarthrosis of the fractured vertebral bodies greatly influences the postoperative height restoration and kyphotic changes after conventional $\mathrm{VP}^{17-20}$; however, the influence of vertebral mobility on the outcomes of KP has been studied very little. ${ }^{21}$ The present study aimed at comparing the postoperative height restoration and kyphotic changes between patients with VCFs undergoing VP and KP, according to the presence or absence of preoperative vertebral mobility.

\section{MATERIALS AND METHODS Subjects}

This study targeted patients with osteoporotic VCFs who visited our hospital between January 2008 and April 2012. We studied 167 vertebral bodies in 119 patients who underwent VP or KP at our hospital because conservative therapy at hospitals in their neighborhood had failed to alleviate their pain. Of those patients, the data pertaining to 34 vertebral bodies in 28 patients treated by 


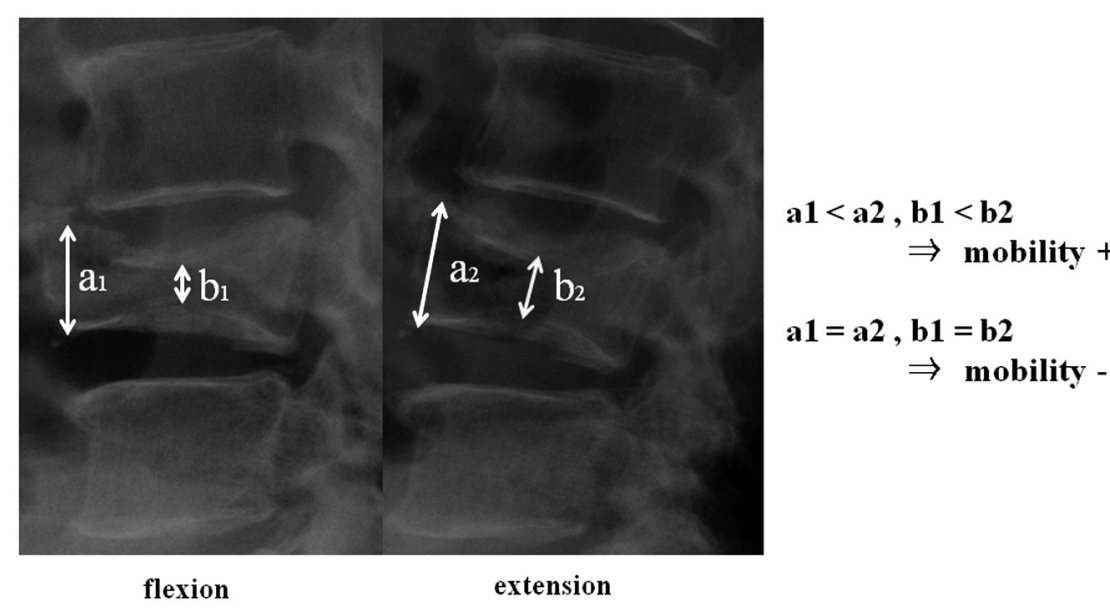

FIG 1. Preoperative lateral radiographs of a patient with VCF with vertebral mobility. The collapsed vertebral body shows dynamic mobility. The vertebral body height increased with extension stress.

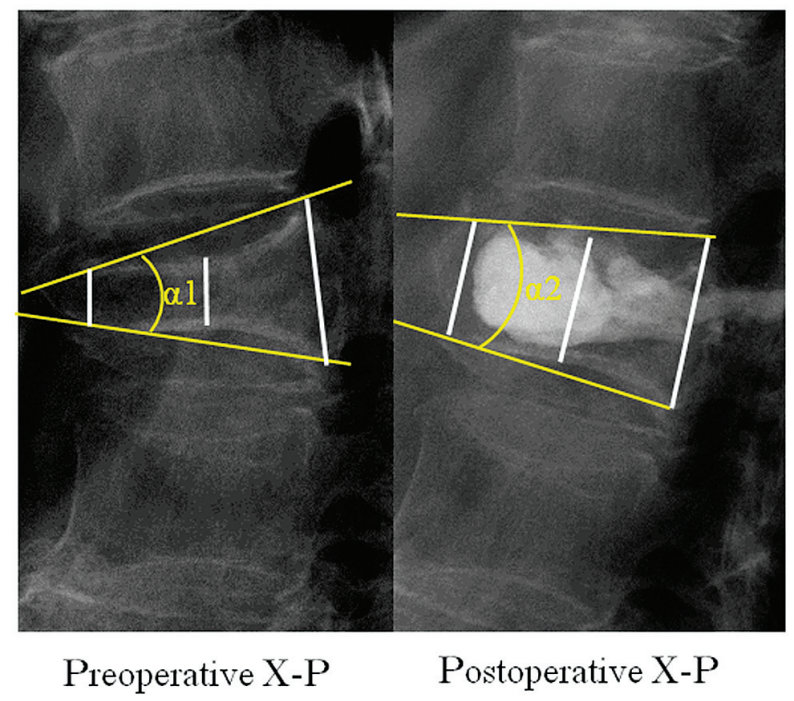

FIG 2. Pre- and postoperative standing lateral radiographs of VCFs. The kyphotic change was defined as the difference between the postoperative kyphotic angle and the preoperative kyphotic angle. We measured the vertebral heights at the anterior, central, and posterior portions of the fractured vertebral bodies and defined vertebral height restoration as the difference between the pre- and postoperative vertebral heights.

VP and 43 vertebral bodies in 38 patients treated by KP who met the criteria described below were included in the present analysis. The patients undergoing VP were treated between January 2008 and August 2009, and those undergoing KP were treated between January 2011 and April 2012. In all patients, simple dynamic lumbar radiographic imaging was performed preoperatively to assess the presence or absence of vertebral mobility. Furthermore, lumbar CT and lumbar MR imaging were performed to determine the indication for surgery. The study protocol was approved by the institutional review board.

\section{Inclusion and Exclusion Criteria}

The inclusion criteria were as follows: 1) VCF with 0\%-90\% loss of vertebral body height on plain $\mathrm{x}$-rays of the spine; 2) severe back pain associated with 1 or 2 VCFs refractory to analgesic med-

\section{Surgical Procedures}

\section{Image Assessment}

ication administered for at least 2 weeks; 3) pain score on a Visual Analog Scale of 5 or higher, interfering with the activities of daily living, or pain on tapping on the spinal process of the fractured vertebra; and 4) the affected vertebral body showing a high signal intensity on short TI inversion recovery MR imaging and a low signal intensity on T1-weighted MR imaging.

The exclusion criteria were as follows: 1) $\geq 3$ vertebral bodies requiring treatment, 2) uncorrected coagulopathy, 3) local or systemic infection, 4) patient not willing to provide informed consent, 5) impaired cardiopulmonary function, 6) spinal metastatic cancer, 7) evidence of radiculopathy, and 8) history of spinal surgery.

Percutaneous Vertebroplasty. All patients were operated on by 1 of the authors (M.K.) with experience in performing $>600$ vertebroplasties. Surgery was performed with the patient under local anesthesia and in the prone position. Under C-arm guidance, a 13-ga biopsy needle was inserted via a transpedicular route on the right or left side into the anterior third of the vertebral body. Contrast medium was injected through the needle, and the position of the tip of the needle and the efflux pattern of the contrast medium were checked. A polymethylmethacrylate mixture was then injected into the vertebral body. When the injected PMMA mixture contacted some part of cortical margin of the vertebral body, the surgery was completed by withdrawing the needle. During the PMMA injection, fluoroscopic monitoring was conducted in both planes with a C-arm unit.

Balloon Kyphoplasty. All patients were operated on by one of the authors (M.K.). In our country, kyphoplasty is accepted by insurance only with the patient under general anesthesia. All surgeries were performed with the patient under general anesthesia and placed in the prone position. In all cases, balloon KP was performed through the transpedicular route on both sides by placement of working cannulas bilaterally by using standard kyphoplasty equipment (Kyphon Xpander; IBT System, Binzwangen, Germany). In the vertebral body, the balloons on each side were inflated to create a space for injection of the bone cement, and then the balloons were deflated and removed. The PMMA (Kyphon HV-R; Medtronic, Leesburg, Virginia) mixture was also injected into the vertebral body from both sides. When the bone cement contacted some part of cortical margin of the vertebral body, the surgery was completed by withdrawing the needles. During the bone cement injection, fluoroscopic monitoring was conducted in both planes with a C-arm unit.

The presence or absence of mobility of the affected vertebral bodies before surgery was assessed by dynamic radiographic imaging. Conventional lateral, lateral flexion, and extension stress radio- 
Table 1: Baseline characteristics of patients treated for VCFs

\begin{tabular}{|c|c|c|c|}
\hline & VP & KP & $P$ Value \\
\hline No. & 28 patients, 34 bodies & 38 patients, 43 bodies & \\
\hline Age (yr) & $74 \pm 8.3$ & $75.5 \pm 6.3$ & .21 \\
\hline $\operatorname{Sex}(M / F)$ & $4: 24$ & $6: 32$ & .58 \\
\hline $\mathrm{TT} 2, \mathrm{~L} 1$ & $23(68 \%)$ & $24(56 \%)$ & .21 \\
\hline Interval (days) & $105.6 \pm 99$ & $75.5 \pm 101$ & .13 \\
\hline Vertebral mobility (+) & 19 (56\%) & $19(44 \%)$ & .21 \\
\hline \multicolumn{4}{|l|}{ Vertebral height(mm) } \\
\hline Anterior & $14.6 \pm 4.6$ & $16.2 \pm 5.5$ & .15 \\
\hline Middle & $12.3 \pm 3.9$ & $13.4 \pm 4.6$ & .32 \\
\hline Posterior & $22.8 \pm 3.6$ & $23.9 \pm 3.6$ & .21 \\
\hline \multicolumn{4}{|l|}{$\mathrm{VAS}^{\mathrm{a}}$} \\
\hline Total & $7.81 \pm 2.5$ & $7.98 \pm 1.7$ & .96 \\
\hline Vertebral mobility $(+)$ & $7.67 \pm 2.4$ & $7.92 \pm 1.6$ & .98 \\
\hline Vertebral mobility $(-)$ & $8.15 \pm 2.6$ & $8.21 \pm 2.0$ & .86 \\
\hline \multicolumn{4}{|c|}{$\begin{array}{l}\text { Note:- }+ \text { indicates positive; }- \text {, negative. } \\
\text { Patients rated their pain on an Visual Analog Scale of } 0=\text { no pain to } 10=\text { severe pain before surgery. }\end{array}$} \\
\hline & VP & KP & $P$ Value \\
\hline \multicolumn{4}{|l|}{ VAS } \\
\hline Total & $1.59 \pm 1.4$ & $2.39 \pm 2.1$ & .20 \\
\hline Vertebral mobility (+) & $1.80 \pm 1.4$ & $2.08 \pm 1.8$ & .84 \\
\hline Vertebral mobility (-) & $1.33 \pm 0.5$ & $2.57 \pm 2.4$ & .24 \\
\hline Cement leak & $16(47.1 \%)$ & $9(21.0 \%)$ & .02 \\
\hline Cement volume $(\mathrm{mL})$ & $3.74 \pm 1.7$ & $2.5 \pm 1.0$ & $<0.01$ \\
\hline Kyphotic change (degree) & $7.0 \pm 5.7$ & $6.9 \pm 7.0$ & .637 \\
\hline \multicolumn{4}{|c|}{ Vertebral height changes (mm) } \\
\hline Anterior & $3.6 \pm 3.4$ & $3.6 \pm 3.5$ & .922 \\
\hline Central & $2.5 \pm 2.3$ & $2.0 \pm 2.2$ & .279 \\
\hline Posterior & $0.9 \pm 1.7$ & $0.5 \pm 0.8$ & .589 \\
\hline
\end{tabular}

Note:-_+ indicates positive; -, negative.

graphs (plain x-rays) were obtained. Vertebral mobility was assessed by comparing the vertebral body heights measured in these images: If there was even a slight difference in the vertebral body height between the lateral flexion and extension images, as shown in Fig 1, vertebral mobility was judged to be present.

The vertebral heights and kyphotic angles of the fractured vertebral bodies were measured in the flexed position on standing lateral radiographs obtained before and after surgery (Fig 2). The vertebral heights were measured in the anterior, central, and posterior portions of the involved vertebrae. Furthermore, the measurements were also conducted in 1 adjacent nonfractured vertebral body on lateral radiographs and CT images to validate the measurements. Vertebral height restoration of the involved vertebrae was defined as the difference between the preoperative and postoperative vertebral height, and the kyphotic change (in degrees) was defined as the difference between the postoperative and preoperative wedge angle. Measurements were performed by the first author (K.Y.) on postoperative radiographs and CT scans obtained on the day after the surgery. First, we compared the vertebral height restoration and kyphotic changes between the VP and KP groups. Then, the patients were divided according to the presence or absence of vertebral mobility on the preoperative images. The vertebral height restorations and kyphotic changes were compared within each treatment group and, furthermore, between the 2 treatment groups according to the presence or absence of vertebral mobility.

Last, the frequency of postoperative bone cement leakage from the vertebral body on postoperative CT scans was analyzed and compared between the 2 treatment groups. The presence of cement leakage into the adjacent intervertebral disk, veins, paravertebral soft tissue, or epidural spaces was defined as any contrast detected beyond the cortical margin of the vertebral body on the postoperative CT scans.

\section{Statistical Analysis}

Statistical analyses were performed by using StatView, Version 5.0 software (SAS Institute, Cary, North Carolina). For comparison of the demographic characteristics, vertebral height restorations, and other factors between the 2 groups, we applied the Mann-Whitney $U$ test or Fisher exact test. All data are presented as mean \pm SD, and differences were considered statistically significant at a $P<.05$.

\section{RESULTS}

The demographic data determined preoperatively in the VP and KP groups are shown in Table 1. No significant differences were observed in the age, sex, location of the treated vertebral bodies, preoperative VAS scores, duration of pain, or preoperative vertebral heights between the 2 groups $(P>.05)$. Preoperative dynamic imaging revealed vertebral mobility in 19 vertebral bodies each in the VP (56\%) and KP (44\%) groups; thus, there was no significant difference $(P=.21)$.

The postoperative results are shown in Table 2. The average vertebral height restoration was $3.63 \pm 3.4 \mathrm{~mm}$ in the VP group and $3.63 \pm 3.5 \mathrm{~mm}$ in the KP group for the anterior portion $(P=$ $.922) ; 2.53 \pm 2.3$ and $2.0 \pm 2.2 \mathrm{~mm}$, respectively, for the central portion $(P=.279)$; and $0.92 \pm 1.7$ and $0.48 \pm 0.8 \mathrm{~mm}$, respectively, for the posterior portion $(P=.589)$. No significant differences were observed in any of the portions between the 2 groups. The average kyphotic change was $7.0^{\circ} \pm 5.7^{\circ}$ in the VP group and $6.9^{\circ} \pm 7.0^{\circ}$ in the KP group, with no significant difference between the 2 groups $(P=.637)$. Then, the patients were divided according to the presence or absence of vertebral mobility, as assessed by preoperative dynamic imaging. The mean vertebral height restoration and kyphotic changes in the anterior portion of the vertebral body were compared in patients with vertebral mobility and in those without it, between the 2 treatment groups, and between the patients with and without vertebral mobility within each of the treatment groups (Figs 3 and 4). The vertebral height restoration and kyphotic changes were significantly better in the patients with unstable vertebral bodies than in those without vertebral instability within each of the treatment groups (vertebral height restoration: VP, $P=.0024 ; \mathrm{KP}, P=.0004$; kyphotic changes: VP, $P=.0046$; KP. $P=.003$ ) (Figs 3 and 4).

On the other hand, comparison of the vertebral height restoration in the anterior portion of the vertebral body in patients 


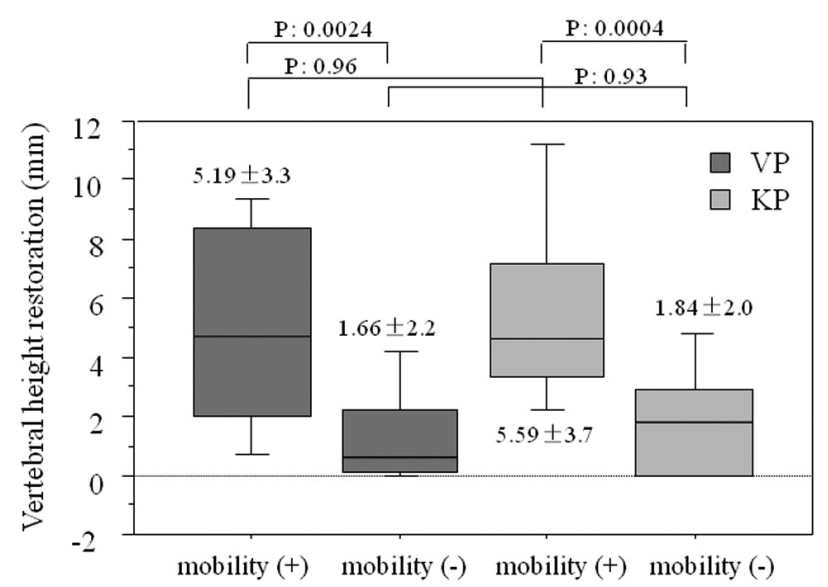

FIG 3. Postoperative vertebral height restoration of the anterior portion.

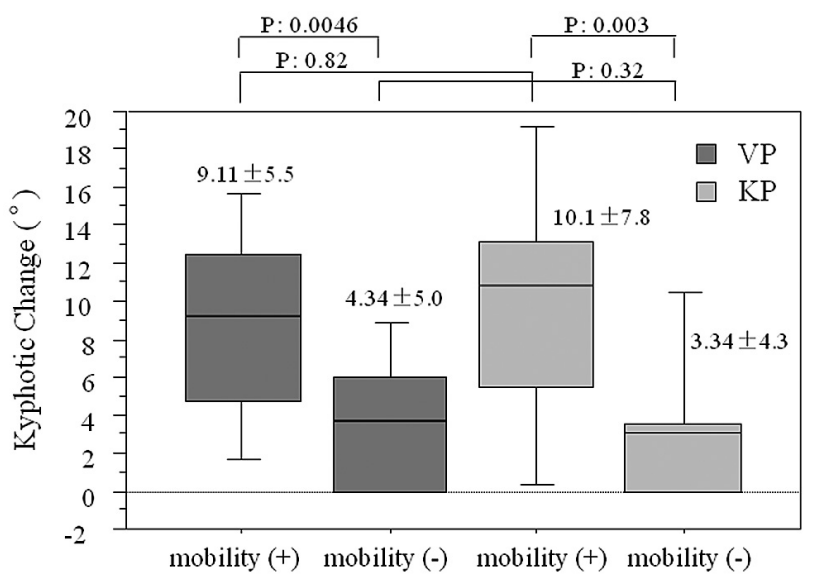

FIG 4. Postoperative kyphotic change of a treated VCF.

with vertebral mobility and those without vertebral mobility between the 2 treatment groups revealed no significant differences (vertebral mobility-positive, $P=.96$; vertebral mobility-negative, $P=.93$ ). Comparison of the kyphotic changes in the patients with vertebral mobility and those without vertebral mobility between the 2 treatment groups also revealed no significant differences (vertebral mobility-positive, $P=.82$; vertebral mobilitynegative, $P=.32$ ). As was the case in the preoperative VAS scores, there was no significant difference in the postoperative VAS scores between the 2 treatment groups. However, the volume of injected bone cement was $3.7 \pm 1.7 \mathrm{~mL}$ in the VP group and $2.5 \pm$ $1.0 \mathrm{~mL}$ in the KP group, being significantly less in the latter $(P<$ $.01)$. Leakage of the injected bone cement was found from 16 vertebral bodies in the VP group (47\%); it was into the disk space in 12 cases. On the other hand, in the KP group, bone cement leaked from 9 vertebral bodies (21\%); in 4 cases, the leakage was into the disk space. Thus, the problem of bone cement leakage from the vertebral body was significantly less in the KP group $(P=.02)$.

\section{DISCUSSION}

It has been reported that the vertebral height restoration achieved with classic VP, without the use of a balloon, is greatly influenced by the presence of vertebral mobility. ${ }^{17-20}$ In cases of VCF with vertebral mobility, the height of the vertebra spontaneously expands due to the prone position during surgery. Accordingly, as this expansion increases the intravertebral vacuum cleft, vertebral height restoration after bone cement injection is favorable. ${ }^{17,18}$ Of course, this phenomenon can also occur in KP. However, there are very few reports on the effects of $\mathrm{KP}$ on vertebral height restoration with preoperative vertebral mobility taken into account. Voggenreiter, ${ }^{21}$ in a study in 30 patients treated by KP for VCFs, measured the changes in the vertebral heights before and after inflation of a balloon and before and after bone cement injection. On the basis of his study, he reported that rather than inflation of a balloon, the vertebral mobility exerted great influence on the postoperative vertebral height restoration.

However, we considered that to verify whether the use of a balloon in KP might actually contribute to the resolution of vertebral deformities, it is necessary to compare the changes in the vertebral heights and kyphotic angles between patients treated by VP and KP after adjustment for preoperative instability of the vertebral body in these patients. No previous study comparing the effects of the 2 procedures on the resolution of the vertebral deformities has taken into consideration preoperative vertebral mobility in patients, to our knowledge. The present study revealed that the presence of vertebral instability exerted a significantly beneficial effect on the resolution of the vertebral deformities after not only VP but also KP. Furthermore, there was no significant difference in the vertebral height restoration or kyphotic changes between the 2 treatment groups either with or without unstable vertebral bodies.

On the basis of the above results, we believe that postoperative vertebral height restoration achieved not only by VP but also KP largely depends on the preoperative vertebral mobility, whereas the effects of use of a balloon itself may be negligible. Therefore, to restore vertebral deformities, one must adjust a patient's position on an operating table precisely during the operation. Actually, we always make an effort to obtain the lumbar extension of patients as much as possible by tuning the operating table finely and skillfully, and inserting a pillow in front of the waist.

Meanwhile, the volume of injected bone cement in the KP group was significantly lower compared with that in the VP group in the present study. This may be attributable to the fact that the diffusion of bone cement in the vertebral body was limited by cavity creation by inflating a balloon, injection of more viscous bone cement, and so forth. The risk of bone cement leakage from the vertebral body will be decreased by weight loss of bone cement. ${ }^{22}$ During both VP and KP, we discontinued injection of bone cement when it approached some part of cortical margin of the fractured vertebral body. During KP, bone cement is injected into the cavity created by the balloon and approaches the posterior wall of the vertebral body along the trajectory of the puncture needle. Thus, bone cement does not diffuse widely in the vertebral body, often resulting in the possibility of injection of only a small volume. However, this effect allows reduction of the risk of bone cement leakage from the vertebral body without compromising the effects on pain relief compared with VP, which may therefore represent one of the merits of KP. Thus, the advantages that may be expected of KP may be the lower volume of bone cement injected and the lower incidence of leakage from the vertebral body, 
rather than better vertebral height restoration or kyphotic changes.

The present study has several limitations. First, we began kyphoplasty for VCFs in January 2011. Therefore, our experience with kyphoplasty is less than that in vertebroplasty; thus, it is a possible cause of the difference in outcome between 2 procedures. Second, our study included only a small number of patients, and the patients were not randomized into the 2 treatment groups; rather they were assigned to the procedures depending on the time of enrollment. VP was performed for those who were enrolled during the first half of the study period, and KP was performed for those who were enrolled during the second half. Thus, despite the lack of randomization, minimal bias might be expected from the assignment of the patients to the procedures. Moreover, because surgery was performed for all patients by the same surgeon at the same institution, there is a good reason to believe that the 2 groups were comparable. Third, the present study was a retrospective one, and vertebral mobility, vertebral heights, and kyphotic angles were not measured and analyzed in a blinded manner. However, the measurements obtained in the present study were consistent with those in previous reports; thus, we believe that there was no problem related to this limitation. Last, on the basis of the analysis in the present study, the volume of injected bone cement during KP was smaller compared with that during VP. This could be a cause for the failure to achieve better resolution of the vertebral deformities following KP. As described above, when the bone cement approached the margin of a vertebral body during KP, we discontinued bone cement injection because we determined that further injection would carry a risk; we did not intentionally decrease the volume of injected bone cement.

\section{CONCLUSIONS}

In order to examine whether inflation of a balloon might actually contribute to the vertebral height restoration or kyphotic changes in $\mathrm{KP}$, comparison with VP was made after adjustment for dynamic fracture mobility. The vertebral height restoration and kyphotic changes that were achieved after surgery largely depended on the preoperative vertebral mobility in not only the patients treated by VP, but also those treated by KP. The use of the balloon in KP contributed little to the resolution of the vertebral deformities following surgery.

\section{REFERENCES}

1. Boonen S, Wahl DA, Nauroy L, et al. Balloon kyphoplasty and vertebroplasty in the management of vertebral compression fractures. Osteoporos Int 2011;22:2915-34

2. Becker S, Pfeiffer KP, Ogon M. Comparison of inpatient treatment costs after balloon kyphoplasty and non-surgical treatment of vertebral body compression fractures. Eur Spine J 2011;20:1259-64

3. Chen L, Yang H, Tang T. Unilateral versus bilateral balloon kyphoplasty for multilevel osteoporotic vertebral compression fractures: a prospective study. Spine 2011;36:534-40

4. Wardlaw D, Cummings SR, Van Meirhaeghe J, et al. Efficacy and safety of balloon kyphoplasty compared with non-surgical care for vertebral compression fracture (FREE): a randomised controlled trial. Lancet 2009;21:1016-24

5. Eck JC, Nachtigall D, Humphreys SC, et al. Comparison of vertebro- plasty and balloon kyphoplasty for treatment of vertebral compression fractures: a meta-analysis of the literature. Spine $J$ 2008;8:488-97

6. Taylor RS, Taylor RJ, Fritzell P. Balloon kyphoplasty and vertebroplasty for vertebral compression fractures: a comparative systematic review of efficacy and safety. Spine (Phila Pa 1976) 2006;31: 2747-55

7. Svedbom A, Alvares L, Cooper C, et al. Balloon kyphoplasty compared to vertebroplasty and nonsurgical management in patients hospitalised with acute osteoporotic vertebral compression fracture: a UK cost-effectiveness analysis. Osteoporos Int 2013;24: 355-67

8. Panagiotis K, Konstantinos V, Thomas R. Balloon kyphoplasty versus KIVA vertebral augmentation: comparison of two techniques for osteoporotic vertebral body fractures-a prospective randomized study. Spine (Phila Pa 1976) 2012 Jul 26. [Epub ahead of print]

9. Ma XL, Xing D, Ma JX, et al. Balloon kyphoplasty versus percutaneous vertebroplasty in treating osteoporotic vertebral compression fracture: grading the evidence through a systematic review and meta-analysis. Eur Spine J 2012;21:1844-59

10. Liu JT, Liao WJ, Tan WC, et al. Balloon kyphoplasty versus vertebroplasty for treatment of osteoporotic vertebral compression fracture: a prospective, comparative, and randomized clinical study. Osteoporos Int 2010;21:359-64

11. Röllinghoff M, Siewe J, Zarghooni K, et al. Effectiveness, security and height restoration on fresh compression fractures: a comparative prospective study of vertebroplasty and kyphoplasty. Minim Invasive Neurosurg 2009;52:233-37

12. Shindle MK, Gardner MJ, Koob J, wt al. Vertebral height restoration in osteoporotic compression fractures: kyphoplasty balloon tamp is superior to postural correction alone. Osteoporos Int 2006;17: 1815-19

13. Li X, Yang H, Tang T, et al. Comparison of kyphoplasty and vertebroplasty for treatment of painful osteoporotic vertebral compression fractures: twelve-month follow-up in a prospective nonrandomized comparative study. J Spinal Disord Tech 2012;25:142-49

14. Zhao YL, Yang HL, Konrad J, et al. Kyphoplasty does not maintain all restored height postoperatively: a prospective, comparative study. Orthopedics 2010; Aug 11;33. doi: 10.3928/0147744720100625-03

15. Hiwatashi A, Westesson PL, Yoshiura T, et al. Kyphoplasty and vertebroplasty produce the same degree of height restoration. AJNR Am J Neuroradiol 2009;30:669-73

16. Dublin AB, Hartman J, Latchaw RE, et al. The vertebral body fracture in osteoporosis: restoration of height using percutaneous vertebroplasty. AJNR Am J Neuroradiol 2005;26:489-92

17. Chen YJ, Chen HY, Tsai PP, et al. Significance of dynamic mobility in restoring vertebral body height in vertebroplasty. AJNR Am J Neuroradiol 2012;33:57-60

18. McKiernan F, Jensen R, Faciszewski T. The dynamic mobility of vertebral compression fractures. J Bone Miner Res 2003;18:24-29

19. Sun G, Jin P, Li M, et al. Height restoration and wedge angle correction effects of percutaneous vertebroplasty: association with intraosseous clefts. Eur Radiol 2011;21:2597-603

20. Teng MM, Wei CJ, Wei LC, et al. Kyphosis correction and height restoration effects of percutaneous vertebroplasty. AJNR Am J Neuroradiol 2003;24:1893-900

21. Voggenreiter G. Balloon kyphoplasty is effective in deformity correction of osteoporotic vertebral compression fractures. Spine 2005;30:2806-12

22. Ryu KS, Park CK, Kim MC, et al. Dose-dependent epidural leakage of polymethylmethacrylate after percutaneous vertebroplasty in patients with osteoporotic vertebral compression fractures. J Neurosurg 2002;96:56-61 